\title{
Glucose Metabolism Disorders in Cirrhosis: Frequency and Risk Factors in Tunisian Population. Results of a Cross-Sectional Study
}

\author{
Rym Ennaifer ${ }^{1,2 *}$, Myriam Cheikh ${ }^{1,2}$, Rania Hefaiedh ${ }^{1,2}$, Hayfa Romdhane ${ }^{1,2}$, \\ Houda Ben Nejma1,2, Najet Bel Hadj1,2 \\ ${ }^{1}$ Department of Hepato-Gastro-Enterology, Mongi Slim Universitary Hospital, Tunis, Tunisia \\ ${ }^{2}$ Faculty of Medicine, University of Tunis El Manar, Tunis, Tunisia \\ Email: rym.ennaifer@yahoo.fr
}

Received 13 June 2014; revised 28 July 2014; accepted 14 July 2014

Copyright (C) 2014 by authors and Scientific Research Publishing Inc.

This work is licensed under the Creative Commons Attribution International License (CC BY).

http://creativecommons.org/licenses/by/4.0/

(c) (i) Open Access

\begin{abstract}
Background and aims: Alterations in carbohydrate metabolism are frequently observed in cirrhosis; to determine the frequency of diabetes mellitus and impaired glucose tolerance in Tunisian cirrhotic patients and identify risk factors. Patients and methods: Cross-sectional study; fasting plasma glucose levels were measured in consecutive patients with cirrhosis. Oral glucose tolerance test was performed if fasting plasma glucose level was normal. Glucose metabolism disorders were then classified as: impaired glucose tolerance and diabetes mellitus. Cirrhotics with glucose metabolism disorder were compared to those without. Results: Seventy-seven patients with cirrhosis were included: $\mathbf{6 8 . 8 \%}$ were diagnosed as having glucose metabolism disorder; diabetes in $\mathbf{4 2 . 8 \%}$ and impaired glucose tolerance in $26 \%$. The tests were able to identify $60.4 \%$ of glucose metabolism disorders. Univariate analysis disclosed a higher proportion of female gender $(p=0.04)$ and more frequent familial history of diabetes mellitus $(p=0.005)$ in the group with glucose metabolism disorder. There were no statistically differences regarding age, etiology and severity of the cirrhosis, and dry body mass index. Multivariate analysis showed that familial history of diabetes was the only independent risk factor $(O R=5.1, p=0.005)$. Conclusion: In our study, the frequency of glucose metabolism disorders was $\mathbf{6 8 . 8 \%}$. Oral glucose tolerance test allowed disclosing nearly half of them, pointing a high incidence of latent glucose metabolism disorders. In this way, it should be routinely evaluated in all patients with cirrhosis. Familial history of diabetes was the only independent risk factor, suggesting that other factors in addition to liver disease may play a role.
\end{abstract}

*Corresponding author.

How to cite this paper: Ennaifer, R., Cheikh, M., Hefaiedh, R., Romdhane, H., Nejma, H.B. and Hadj, N.B. (2014) Glucose Metabolism Disorders in Cirrhosis: Frequency and Risk Factors in Tunisian Population. Results of a Cross-Sectional Study. Open Journal of Gastroenterology, 4, 289-294. http://dx.doi.org/10.4236/ojgas.2014.48042 


\section{Keywords}

\section{Cirrhosis, Diabetes, Impaired Glucose Tolerance}

\section{Introduction}

Glucose metabolism disorders (GMD) are frequent in liver cirrhosis [1]. The liver plays a key role in blood glucose control, thus, in the presence of chronic liver disease, the metabolic homeostasis of glucose is impaired and results in glucose intolerance and diabetes mellitus (DM) type 2 [2]. There is a wide variability in the prevalence of DM and impaired glucose tolerance (IGT) according to the literature [3]. About 50\% - 80\% of cirrhotic patients have IGT and 30\% - 40\% develop DM [4]. Moreover, DM in cirrhosis may be subclinical, since fasting serum glucose may be normal. In these cases, it is necessary to perform an oral glucose tolerance test (OGTT) to detect an impairment of glucose metabolism [2]. Previous studies have shown that the DM increases the risk of complications of cirrhosis and reduces survival [5]. Risk factors for GMD in cirrhosis have been thoroughly investigated.

This study is the first one to determine the prevalence of DM and IGT in Tunisian cirrhotic patients and identify factors that might be potentially associated with GMD.

\section{Patients and Methods}

\subsection{Patients}

From August 2011 to July 2012, we consecutively included patients with cirrhosis admitted to the department of Gastroenterology and Hepatology of Mongi Slim University Hospital in Tunis. Inclusion criteria were: presence of cirrhosis diagnosed according to histological analysis (Fibrosis grade 4 of METAVIR) or a combination of clinical criteria (splenomegaly, ascitis, collateral venous circulation), laboratory tests (decreased prothrombin and factor V levels, hypoalbuminemia, hypocholesterolemia) and imaging (portal venous dilatation, ascitis, splenomegaly on ultrasound and endoscopic portal hypertension findings); age above 18 years. Exclusion criteria were: patients with chronic pancreatitis, chronic ingestion of corticosteroids, diabetes type 1 , others specific types of diabetes and gestational diabetes. Patients with autoimmune hepatitis receiving corticosteroids were also excluded from this study.

\subsection{Methods}

Age, sex, familial history of DM were recorded. We could not measure insulin resistance because it was not available in our center. Complications of cirrhosis (variceal bleeding, encephalopathy, ascites, infections, hepatocellular carcinoma) were assessed. Child-Pugh and Meld scores were calculated. Dry body mass index (dry BMI) was calculated using the standard formula with dry weight in order to compare among patients with and without ascites and/or oedemas [6]. Patients receiving therapy with insulin or oral hypoglycemic medications were considered as diabetics. Other patients were tested for fasting plasma glucose (FPG) twice on different days after 12 hours overnight fast, and an OGTT was performed to patients with normal FPG (less than 5.6 $\mathrm{mmol} / \mathrm{l})$. The OGTT was performed as follows, according to the WHO criteria: patients fasted for at least $12 \mathrm{~h}$, baseline glucose level was measured; they were given $75 \mathrm{~g}$ of oral glucose load, and after $2 \mathrm{~h}$ a second sample was taken (2 h PG).

According to the latest American Diabetes Association criteria (ADA) [7]:

- $\quad$ DM was diagnosed if FPG $\geq 126 \mathrm{mg} / \mathrm{dl}(7 \mathrm{mmol} / \mathrm{l})$ or $2 \mathrm{~h} \mathrm{PG} \geq 200 \mathrm{mg} / \mathrm{dl}(11.1 \mathrm{mmol} / \mathrm{l})$.

- IGT was diagnosed if $2 \mathrm{~h}$ PG was between $140(7.8 \mathrm{mmol} / \mathrm{l})$ and $199 \mathrm{mg} / \mathrm{dl}(11 \mathrm{mmol} / \mathrm{l})$.

The study protocol conformed to the ethical guidelines of the 1975 Declaration of Helsinki as approval by the institution's review board.

\subsection{Statistical Analysis}

Analysis was performed using SPSS version 19. Results were expressed as mean +/- standard deviation. An univariate analysis searching for the factors possibly associated with GMD was firstly performed by comparison 
between cirrhotic with and without GMD using Student $t$ test for continuous variables and the Chi-square or Fisher exact test for categorical variables. Then, a multivariate analysis based on a stepwise logistic regression model was used to assess the independent effect of variables found significant at the univariate analysis. A p-value of less than 0.05 was considered statistically significant.

\section{Results}

\subsection{Patient Population}

Seventy-seven patients (42 females, 35 males) with cirrhosis were included in the study. They had a mean age of $57.1 \pm 13.8$ years (range: 18 - 84). The main etiologies of liver disease were hepatitis $C$ in 28 patients, hepatitis $\mathrm{B}$ in 15 patients and autoimmune in 14 patients. The median duration of liver cirrhosis was 36 months (range: 0 - 240 months). Regarding the severity of the liver disease, 49 patients were classified as Child-Pugh B and C and the mean Meld score was $14.5 \pm 6$ (range: 5 - 33). History of ascitic decompensation (76.6\%), hepatic encephalopathy (37.7\%) and bacterial infections (spontaneous peritoneal infection, urinary infection and bronchopulmonary infection) in 51.9\% were the most frequent complications related to cirrhosis. Demographical and clinical characteristics are given in Table 1, Table 2 and Table 3.

\subsection{Frequency of GMD}

Fifty-three (68.8\%) patients were diagnosed as having GMD: DM in 33 patients (42.8\%) and ITG in 20 patients (26\%). The OGTT was able to identify 25 GMD (47.1\%): 20 ITG and 5 DM. Among patients with DM, 12 (36.3\%) were diagnosed at the same time or after the diagnosis of cirrhosis so they may be considered as having hepatogenous diabetes. Figure 1 summarizes these findings.

\subsection{Comparison between Patients with and without GMD}

- Univariate analysis disclosed a higher proportion of female gender $(p=0.04)$ and more frequent familial history of DM $(\mathrm{p}=0.005)$ in the group with GMD.

There were no statistically differences regarding age, etiology (viral/non viral; cryptogenic/non-cryptogenic) of the cirrhosis, Child-Pugh and Meld scores, mean duration of liver disease, cirrhosis complications and dry BMI.

- Multivariate analysis showed that family history of diabetes was the only independent risk factor for GMD in cirrhosis: $\mathrm{OR}=\mathbf{5 . 1}, \mathrm{CI}=[\mathbf{1 . 6}-\mathbf{1 6}], \mathrm{p}=\mathbf{0 . 0 0 5}$.

Table 4 and Table 5 summarize these findings.

Table 1. Demographical and clinical characteristics of patients.

\begin{tabular}{cc}
\hline Characteristics & $\mathrm{N}=77$ \\
Age (y) & $57.1 \pm 13.8(18-84)$ \\
Sex (females) & $42(54.5 \%)$ \\
Child-Pugh score & $\mathrm{A}=28(36.3 \%), \mathrm{B}=27(35.1 \%), \mathrm{C}=22(28.6 \%)$ \\
Meld score & $14.5 \pm 6(5-33)$ \\
\hline
\end{tabular}

Table 2. Cirrhosis etiology.

\begin{tabular}{llc}
\hline Cirrhosis etiology & $\mathrm{N}=77$ \\
& - HCV & $28(36.3 \%)$ \\
& - HBV & $15(19.5 \%)$ \\
& - Auto-immune & $14(18.2 \%)$ \\
& - Cryptogenic & $11(14.3 \%)$ \\
- & Alcohol & $3(3.9 \%)$ \\
- & NASH & $1(1.3 \%)$ \\
- & Others & $5(6.4 \%)$ \\
\hline
\end{tabular}

HCV: hepatitis C virus; HBV: hepatitis B virus; NASH: non-alcoholic steato-hepatitis. 
Table 3. Cirrhosis complications.

\begin{tabular}{llc}
\multicolumn{2}{l}{ Cirrhosis complications } & \\
- & Ascitic decompensation & $59(76.6 \%)$ \\
- & Oesophageal varices & $59(76.6 \%)$ \\
- & Bleeding oesophageal varices & $27(35.1 \%)$ \\
- & Refractory ascites & $14(18 \%)$ \\
- & Hepatic encephalopathy & $29(37.7 \%)$ \\
- & Hepatocellular carcinoma & $17(22.1 \%)$ \\
- & Bacterial infections & $40(51.9 \%)$
\end{tabular}

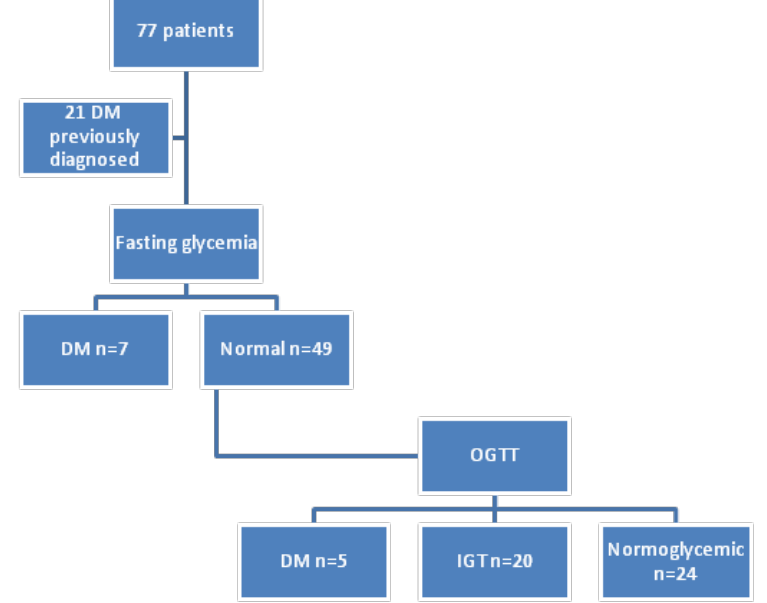

Figure 1. Results according to fasting glycemia and OGTT in the studied patients (DM: diabetes mellitus, OGTT: oral glucose tolerance test, IGT: impaired glucose tolerance).

Table 4. Clinical and epidemiological characteristics of cirrhotic patients with and without GMD.

\begin{tabular}{|c|c|c|c|c|}
\hline & Cirrhosis with GMD $(\mathrm{n}=53)$ & Cirrhosis without GMD $(n=24)$ & $\mathrm{P}$ & OR [CI 95\%] \\
\hline Age & 56 & 58 & 0.58 & \\
\hline Female gender & $33(62 \%)$ & $9(37.5 \%)$ & 0.04 & $1.8[0.5-6]$ \\
\hline Dry BMI $\left(\mathrm{kg} / \mathrm{m}^{2}\right)$ & 25 & 23 & 0.19 & \\
\hline Familial history of diabetes & $30(56.6 \%)$ & $6(25 \%)$ & 0.005 & 3.84 [1.2 - 13] \\
\hline Child Pugh score B or C & $35(66 \%)$ & $(58.4 \%)$ & 0.51 & \\
\hline Mean Meld score & 14 & 15 & 0.45 & \\
\hline Etiology of cirrhosis & & & & \\
\hline $\begin{array}{ll}- & \text { Viral/non viral } \\
\text { - } & \text { Cryptogenic/non } \\
& \text { cryptogenic }\end{array}$ & $\begin{array}{c}30 / 23 \\
8 / 45\end{array}$ & $\begin{array}{c}13 / 11 \\
4 / 20\end{array}$ & $\begin{array}{l}\text { NS } \\
\text { NS }\end{array}$ & \\
\hline
\end{tabular}

BMI: body mass index, GMD: glucose metabolism disorders.

Table 5. Cirrhosis complications in patients with and without GMD.

\begin{tabular}{|c|c|c|c|}
\hline Cirrhosis complications & Patients with GMD & Patients without GMD & $\mathrm{P}$ \\
\hline - $\quad$ Ascitic decompensation & $77.7 \%$ & $75 \%$ & 0.82 \\
\hline - $\quad$ Hepatic encephalopathy & $37.7 \%$ & $37.5 \%$ & 0.98 \\
\hline - $\quad$ Bleeding oesophageal varices & $36.5 \%$ & $33.3 \%$ & 0.78 \\
\hline - $\quad$ Hepatocellular carcinoma & $21 \%$ & $25 \%$ & 0.67 \\
\hline - $\quad$ Bacterial infections & $54.8 \%$ & $45.8 \%$ & 0.47 \\
\hline
\end{tabular}




\section{Discussion}

IGT and DM are both frequently prevalent in cirrhosis. According to the literature, 50\% - $80 \%$ of cirrhotic patients and even up to 96\% have IGT while DM occurs in 30\% - 40\% [4] [8]. In most published studies, subclinical GMD are not routinely identified with OGTT, so the magnitude of the problem is often underestimated [8].

Our study is relevant because it prospectively assessed overt and subclinical GMD in a Tunisian population. We disclosed $68.8 \%$ of GMD: $42.8 \%$ of DM and 26\% of IGT, which is about 4 times more than in the Tunisian general population (9.9\% vs 42.8\%), indicating that patients with cirrhosis are a high-risk population for GMD, although comparison is not adequate because the design of the 2 studies is different [9]. In our series, nearly half of GMD were subclinical and identified with OGTT, justifying its use as a routine in practice for this population. In our cohort, the greater prevalence of DM than described in previous studies may be related to the use of OGTT or because our patients had a more severe disease, although we did not found association between severity of liver disease using Child-Pugh and Meld scores. This finding does not agree with previous studies showing that the incidence of DM increases as liver function deteriorates [2] [6] [10].

It has been speculated that genetic and environmental factors and etiology of the liver disease, such hepatitis $\mathrm{C}$ and alcohol impair the insulin secretion [2]. Despite a high proportion of hepatitis $\mathrm{C}$ in our series, we did not found a statistically significant higher frequency of HVC in cirrhotic patients with GMD.

Several parameters are considered as possible risk factors associated with insulin resistance and DM in the general population, including age, female sex, family history of diabetes and overweight [10] [11]. However, in patients who present DM after the development of liver cirrhosis represent a different entity, called hepatogenous diabetes, classical risk factors are less frequently elicited, but this entity is not recognized by the ADA and the WHO [2]. In our study we could not always determine exactly if the onset of DM was before or after liver disease, firstly because in our population, cirrhosis is often diagnosed at the stage of complications, secondly because DM may be subclinical. In concordance with clinical characteristics of hepatogenous diabetes, we did not find a significant difference for age and BMI between diabetics and non diabetics cirrhotic patients, although weight was estimated subtracting ascites and oedema weight. On the other hand, familial history of diabetes was the only independent predictive factor for DM in cirrhosis, suggesting that they are possible adjunctive factor to liver disease in the arisen of DM. This is in agreement with the study of Zein et al. suggesting that cirrhosis alone do not always induce diabetes, and the cause of liver disease as well as environmental factors may play a role [12].

Retrospective studies have shown that DM (either type 2 or hepatogenous) increases the risks of complications of cirrhosis and reduces survival. Indeed, DM accelerates liver fibrosis and inflammation and enhance incidence of bacterial infection [5]. In our study, DM was not associated with a higher prevalence of cirrhosis complications. These results may be related to high rate of subclinical DM which may less contribute to liver injury.

Finally, it has been reported that hepatogenous diabetes is less associated with retinopathy, cardiovascular and renal complications and more frequently associated with hypoglycemic episodes as a result of impaired liver function. Liver disease abnormalities (low intravascular coagulability, low cholesterol, lower prevalence of hypertension) as well as shorter duration of DM may explain relatively lower rate of diabetic complication in chronic liver disease [13] [14].

The strength of our study is that it is the first one carried on a Tunisian population, allowing a preliminary determination of the prevalence and risk factors of GMD in cirrhosis and its design cross-sectional.

Nevertheless, our study had limitations:

1) In patients previously diagnosed as having DM, we could not clearly determine the chronology of the installation of diabetes in order to distinguish between DM type 2 and hepatogenous diabetes.

2) We did not determine the impact of the duration of cirrhosis on the prevalence of DM.

3) We did not include a control group without cirrhosis to compare the incidence of diabetes complications, but this was not the major aim of the study.

\section{Conclusion}

In our study, the frequency of GMD was 68.8\%, with a majority of DM. OGTT disclosed nearly half of GMD, pointing a high incidence of latent GMD in cirrhosis. In this way, it should be routinely evaluated in all patients with cirrhosis. Familial history of diabetes was the only independent risk factor for GMD, suggesting that other 
factors in addition to liver disease may play a role in the development of GMD in cirrhosis.

\section{References}

[1] Buyse, S. and Valla, D. (2007) Carbohydrate Metabolism Dysregulation in Cirrhosis: Pathophysiology, Prognostic Impact and Therapeutic Implications. Gastroentérologie Clinique et Biologique, 31, 266-273. http://dx.doi.org/10.1016/S0399-8320(07)89371-7

[2] Garcia-Compean, D., Jaquez-Quintana, J.O., Gonzales-Gonzales, J.A. and Maldonado-Garza, H. (2009) Liver Cirrhosis and Diabetes: Risk Factors, Pathophysiology, Clinical Implications and Management. World Journal of Gastroenterology, 15, 280-288. http://dx.doi.org/10.3748/wjg.15.280

[3] Alavian, S.M., Hajarizadeh, B., Nematizadeh, F. and Larijani, B. (2004) Prevalence and Determinants of Diabetes Mellitus among Patients with Chronic Liver Disease. BMC Endocrine Disorders, 4, 4. http://dx.doi.org/10.1186/1472-6823-4-4

[4] Wlazlo, N., Van Greevenbroek, M.M., Curvers, J., Schoon, E.J., Friederich, P., Twisk, J.W., et al. (2013) Diabetes Mellitus at the Time of Diagnosis of Cirrhosis Is Associated with Higher Incidence of Spontaneous Bacterial Peritonitis, but Not with Increased Mortality. Clinical Science, 125, 341-348. http://dx.doi.org/10.1042/CS20120596

[5] Jaquez Quintana, J.O., Garcia-Compean, D., Gonzales Gonzales, J.A., Villarreal Perez, J.Z., Lavalle Gonzales, F.J., Munoz Espinosa, L.E., et al. (2011) The Impact of Diabetes Mellitus in Mortality of Patients with Compensated Liver Cirrhosis-A Prospective Study. Annals of Hepatology, 10, 56-62.

[6] Bragança, A.C. and Alvares-da-Silva, M.R. (2010) Prevalence of Diabetes Mellitus and Impaired Glucose Tolerance in Patients with Decompensated Cirrhosis Being Evaluated for Liver Transplantation: The Utility of Oral Glucose Tolerance Test. Archives of Gastroenterology, 47, 22-27.

[7] American Diabetes Association (2011) Diagnosis and Classification of Diabetes Mellitus. Diabetes Care, 34, S62-S69. http://dx.doi.org/10.2337/dc11-S062

[8] Garcia-Compean, D., Jaquez-Quintana, J.O., Lavalle-Gonzales, F.J., Reyes-Cabello, E., Gonzales-Gonzales, J.A., Munoz-Espinosa, L.E., et al. (2012) The Prevalence and Clinical Characteristics of Glucose Metabolism Disorders in Patients with Liver Cirrhosis. A Prospective Study. Annals of Hepatology, 11, 240-248.

[9] Bouguerra, R., Alberti, H., Salem, L.B., Rayana, C.B., Atti, J.E., Gaigi, S., et al. (2007) The Global Diabetes Pandemic: The Tunisian Experience. European Journal of Clinical Nutrition, 61, 160-165. http://dx.doi.org/10.1038/sj.ejcn.1602478

[10] Gentile, S., Loguercio, C., Marmo, R., Carbone, L. and Del Vecchio Blanco, C. (1993) Incidence of Altered Glucose Tolerance in Liver Cirrhosis. Diabetes Research and Clinical Practice, 2, 37-44. http://dx.doi.org/10.1016/0168-8227(93)90130-W

[11] Hickman, I.J. and Macdonald, G.A. (2007) Impact of Diabetes on the Severity of Liver Disease. The American Journal of Medicine, 120, 829-834. http://dx.doi.org/10.1016/j.amjmed.2007.03.025

[12] Zein, N.N., Abdulkarim, A.S., Wiesner, R.H., Egan, K.S. and Persing, D.H. (2000) Prevalence of Diabetes Mellitus in Patients with End-Stage Liver Cirrhosis Due to Hepatitis C, Alcohol, or Cholestatic Disease. Journal of Hepatology, 32, 209-217. http://dx.doi.org/10.1016/S0168-8278(00)80065-3

[13] Vidal, J., Ferrer, J.P., Esmatjes, E., Salmeron, J.M., Gonzales-Clemente, J.M., Gomis, R., et al. (1994) Diabetes Mellitus in Patients with Liver Cirrhosis. Diabetes Research and Clinical Practice, 25, 19-25. http://dx.doi.org/10.1016/0168-8227(94)90157-0

[14] Moscatiello, S., Manini, R. and Marchesini, G. (2007) Diabetes and Liver Disease: An Ominous Association. Nutrition, Metabolism and Cardiovascular Diseases, 17, 63-70. http://dx.doi.org/10.1016/j.numecd.2006.08.004 
Scientific Research Publishing (SCIRP) is one of the largest Open Access journal publishers. It is currently publishing more than 200 open access, online, peer-reviewed journals covering a wide range of academic disciplines. SCIRP serves the worldwide academic communities and contributes to the progress and application of science with its publication.

Other selected journals from SCIRP are listed as below. Submit your manuscript to us via either submit@scirp.org or Online Submission Portal.
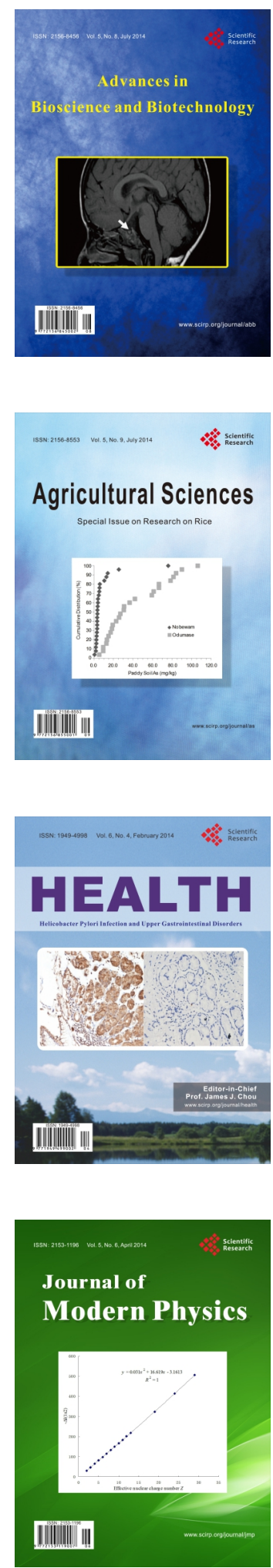
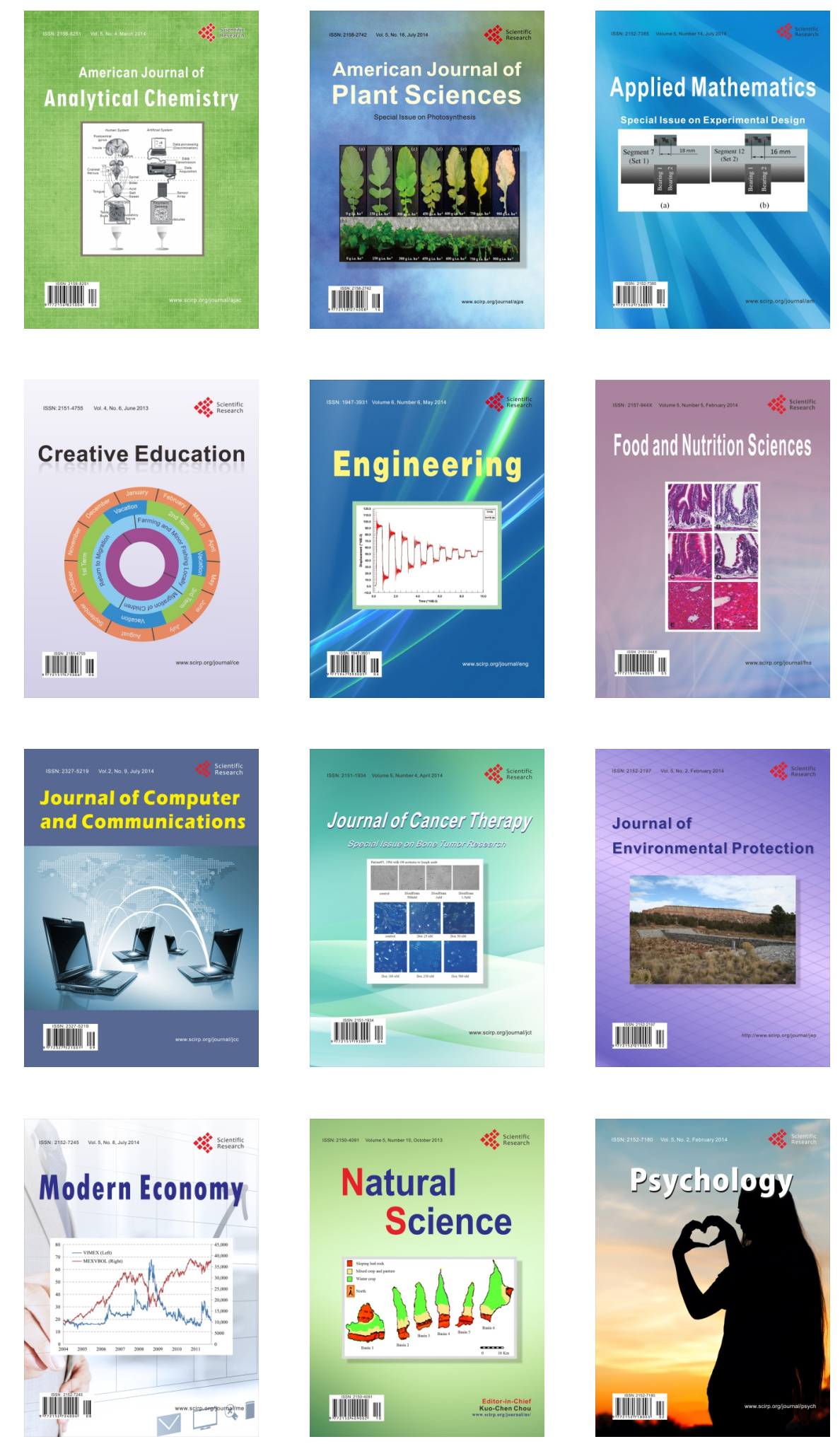